\title{
Closed-Form Solutions of the Thomas-Fermi in Heavy Atoms and the Langmuir-Blodgett in Current Flow ODEs in Mathematical Physics
}

\author{
Efstathios E. Theotokoglou, Theodoros I. Zarmpoutis, and Ioannis H. Stampouloglou \\ School of Applied Mathematical and Physical Sciences, Department of Mechanics, Laboratory of Testing and Materials, \\ National Technical University of Athens, Zographou Campus, Theocaris Building, 5 Heroes of Polytechniou Avenue, \\ 157-73 Athens, Greece \\ Correspondence should be addressed to Efstathios E. Theotokoglou; stathis@central.ntua.gr
}

Received 25 March 2015; Revised 2 July 2015; Accepted 7 July 2015

Academic Editor: Hang Xu

Copyright (C) 2015 Efstathios E. Theotokoglou et al. This is an open access article distributed under the Creative Commons Attribution License, which permits unrestricted use, distribution, and reproduction in any medium, provided the original work is properly cited.

\begin{abstract}
Two kinds of second-order nonlinear, ordinary differential equations (ODEs) appearing in mathematical physics are analyzed in this paper. The first one concerns the Thomas-Fermi (TF) equation, while the second concerns the Langmuir-Blodgett (LB) equation in current flow. According to a mathematical methodology recently developed, the exact analytic solutions of both TF and LB ODEs are proposed. Both of these are nonlinear of the second order and by a series of admissible functional transformations are reduced to Abel's equations of the second kind of the normal form. The closed form solutions of the TF and LB equations in the phase and physical plane are given. Finally a new interesting result has been obtained related to the derivative of the TF function at the limit.
\end{abstract}

The paper is dedicated to the memory of the authors' Professor in National Technical University of Athens Dr. D. E. Panayotounakos who contributed a lot to the study of Thomas-Fermi and Langmuir-Blodgett equations

\section{Introduction}

An equation of considerable interest, called the LangmuirBlodgett (LB) equation $[1,2]$, appeared in connection with the theory of flow of a current from a hot cathode to a positively charged anode in a high vacuum. The cathode and anode are long coaxial cylinders. It was proved [2, page 409] that the LB equation has an analytic expansion in the neighborhood of a fixed value of the independent variable, which assumes arbitrarily given values of the dependent variable and its derivative provided the zero value. However, the above equation does not admit exact analytic solution in terms of known tabulated functions [3, 4]. A lot of applications have been proposed for Langmuir equation. Horváth et al. [5] used Martin-Synge algorithm or anti-Langmuir in liquid chromatography, Shang and Zheng [6] proposed the system of
Zakharov equations which involves the interaction between Langmuir and ion-acoustic waves in plasma, Graham and Cairns [7] posed the constraints on the formation and structure of Langmuir eigenmodes in the solar wind, and so forth.

On the other hand, the Thomas-Fermi (TF) equation appears in the problem of determining the effective nuclear charge in heavy atoms $[2,8,9]$. Numerous applications have also been proposed for TF equation. Tsurumi and Wadati [10] applied the TF approximation to obtain the dynamics of magnetically trapped boson-fermion, Liao [11] proposed an analytic technique for TF equation, Abbasbandy and Bervillier [12] used analytic continuation of Taylor series to confront the TF equation, Ourabah and Tribeche [13] presented a TF model based on thermal nonextensive relativistic effects, and so forth. 
Both LB and TF equations do not admit exact analytic solution in terms of known (tabulated) functions. In this paper using series of admissible functional transformations, at first both $\mathrm{LB}$ and TF nonlinear ODEs are reduced to equivalent Abel's equations of the second kind of the normal form. According to a mathematical methodology recently developed [14-16], we extract closed-form solution of the above Abel equations in both phase and physical planes under initial data in accordance with the physical problems.

The mathematical technique introduced in our study is general and can be applied to a very large class of yet unsolvable ODEs in nonlinear mechanics and generally in mathematical physics. The method employed is exact in accordance with the boundary conditions of each problem under consideration and gives us the possibility not to consider the a priori approximate construction of the solutions in the form of power series, some coefficient of which must be estimated. In addition with our analysis the exact value of the first order derivative of Thomas Fermi equation at $x=0$ is derived in a different way from the methods already existing in the literature [17-21].

\section{Preliminaries: Notations}

A nonlinear ODE of considerable interest, called the Langmuir-Blodgett (LB) equation [1], is the following:

$$
3 y y_{x x}^{\prime \prime}+y_{x}^{\prime 2}+4 y y_{x}^{\prime}+y^{2}-1=0 .
$$

Here the notations $y_{x}^{\prime}=d y / d x, y_{x x}^{\prime \prime}=d^{2} y / d x^{2}, \ldots$ are used for the total derivatives.

This equation of constant coefficients appeared in connection with the theory of flow of a current from a hot cathode to a positive charged anode in a high vacuum. The cathode and anode are long coaxial cylinders and the independent variable $y$ is defined by the equation: $y=f\left(r / r_{0}\right)$, where $r$ is the radius of the anode enclosing a cathode of radius $r_{0}$. The independent variable $x$ is given by $x=\ln \left(r / r_{0}\right)$.

Equation (1) is converted into a somewhat more tractable form by means of the transformation $y=e^{-x / 2} z(x)$ proposed by Davis [2] and thus becomes

$$
3 z z_{x x}^{\prime \prime}+z_{x}^{\prime 2}-e^{x}=0 .
$$

Numerical techniques concerning the solution of (1) are included in [6].

The TF nonlinear ODE

$$
y_{x x}^{\prime \prime}=x^{-1 / 2} y^{3 / 2}
$$

appears in the problem of determining the effective nuclear charge in heavy atoms $[8,9]$. The solution is defined for the boundary values

$$
\begin{gathered}
y(0)=1, \\
y(\infty)=0 .
\end{gathered}
$$

Interesting approximate and numerical integrations methods have been proposed by Sommerfeld [22], Bush and Caldwell [17], Kobayashi et al. [20], Feynman et al. [18], and Coulson and March [19].
The differential equation (3) belongs to equations of Emden-Fowler type $[3,4]$. Taking into account the known exact parametric solutions of this class of nonlinear ODEs [4, pages 241-250], the above mentioned equation can not solve analytically in terms of known functions.

Many investigations have studied the TF equation obeying to the boundary conditions (4) (see [18-21]) and they carried out the outward numerical integration $(x=0, y \rightarrow$ $\infty$ ) for a sequence of values of initial slope converging step by step to that curve which should approach the $x$-axis asymptotically. Finally for a semianalytical solution methodology we must refer to Sommerfeld [22].

The Emden-Fowler nonlinear ODE of the normal form is

$$
y_{x x}^{\prime \prime}=A x^{n} y^{m}
$$

where $A$ and $n, m$ are arbitrary parameters. We note the following regarding the reduction of (5) (see [4]).

For $m \neq 1$ and $m \neq-2 n-3$ the admissible functional transformations

$$
\begin{aligned}
& \xi=\frac{2 n+m+3}{m-1} x^{(n+2) /(m-1)} y \\
& u=x^{(n+2) /(m-1)}\left(x y_{x}^{\prime}+\frac{n+2}{m-1} y\right)
\end{aligned}
$$

reduce the nonlinear ODE (5) to the following Abel equation of the second kind of the normal form

$$
\begin{aligned}
u u_{\xi}^{\prime}-u= & -\frac{(n+2)(n+m+1)}{(2 n+m+3)^{2}} \xi \\
& +A\left(\frac{m-1}{2 n+m+3}\right)^{2} \xi^{m} .
\end{aligned}
$$

In what follows, by a series of admissible functional transformations, we prove that both of (1) and (3) can be exactly reduced to Abel's equations of the second kind of the normal form $y y_{x}^{\prime}-y=f(x)$. According to the following reduction procedure the exact analytic solutions of the above Abel equations are constructed, avoiding the approximate expression of the solutions in power series.

\section{The Reduction Procedure}

3.1. The LB Equation. The LB equation (1) is a second order nonlinear ODE of constant coefficients. Thus, by the substitution

$$
\begin{aligned}
y_{x}^{\prime} & =p(y) \\
& \Longrightarrow y_{x x}^{\prime \prime}=p_{y}^{\prime} p,
\end{aligned}
$$

it can be reduced to the following Abel equation of the second kind:

$$
3 y p p_{y}^{\prime}=-p^{2}-4 y p-y^{2}+1
$$

Furthermore, the well-known transformation [4, page 50]

$$
\begin{aligned}
w(y) & =p e^{\int(1 / 3 y) d y}=p \exp \left(\ln |y|^{1 / 3}\right)=y^{1 / 3} p \\
& \Longrightarrow p=y^{-1 / 3} w ; \quad y \neq 0,
\end{aligned}
$$


giving the expressions

$$
\begin{gathered}
p_{y}^{\prime}=y^{-1 / 3} w_{y}^{\prime}-\frac{1}{3} y^{-4 / 3} w \\
p p_{y}^{\prime}=y^{-2 / 3} w w_{y}^{\prime}-\frac{1}{3} y^{-5 / 3} w^{2},
\end{gathered}
$$

transforms (9) into the following Abel equation of the second kind:

$$
w w_{y}^{\prime}=-\frac{4}{3} y^{1 / 3} w+\frac{1}{3} y^{-1 / 3}-\frac{1}{3} y^{5 / 3}
$$

Finally, the substitution

$$
\begin{aligned}
w(y) & =n(\xi), \\
\xi & =\frac{4}{3} \int y^{1 / 3} d y \\
& \Longrightarrow y=\xi^{3 / 4}
\end{aligned}
$$

reduces (12) to the following Abel equation of the second kind of the normal form:

$$
n n_{\xi}^{\prime}+n=\frac{1}{4} \xi^{-1 / 2}-\frac{1}{4} \xi
$$

or, setting

$$
n(\xi)=-z(\xi)
$$

to the form

$$
z z_{\xi}^{\prime}-z=\frac{1}{4} \xi^{-1 / 2}-\frac{1}{4} \xi
$$

The solution of (16), and thus the solutions of (14), (12), and (9), constitutes the intermediate integral of the LB equation (1) in the phase plane. In other words, when obtaining the solution of the transformed equation (16) in the form $z=z(\xi, \stackrel{*}{C}), \stackrel{*}{C}=$ first integration constant, the solution of (14) becomes $n(\xi)=-z(\xi)$, the solution of $(12)$ is $w(y)=-z\left(y^{4 / 3}\right)$, and finally the solution of $(8)$ is $p(y)=-y^{-1 / 3} z\left(y^{4 / 3}\right)$. Thus, the solution to the original Langmuir equation (1) in the physical plane can be obtained by the integration by parts of the following equation:

$$
\begin{aligned}
& \frac{d y}{p(y)}=d x \\
& \Longrightarrow \int \frac{d y}{y^{1 / 3} z\left(y^{4 / 3}\right)}=-x+C \\
& C=\text { second integration constant. }
\end{aligned}
$$

Note that the reduced Abel equation (16) does not admit an exact analytic solution in terms of known (tabulated) functions [4, pages 29-45].

3.2. The TF Equation. Equation (3) is a typical Emden-Fowler nonlinear equation with $A=1, n=-1 / 2$, and $m=3 / 2$. A thorough examination concerning approximate expansion solution of (3) is included in [20] as well as all the references cited there. By now, with the aid of the transformations (6) one reduces the original TF equation (3) to the following Abel equation of the second kind of the normal form:

$$
u u_{\xi}^{\prime}-u=-\frac{12}{49} \xi+\frac{1}{49} \xi^{3 / 2}
$$

where

$$
\begin{aligned}
& \xi=7 x^{3} y, \\
& u=x^{3}\left(x y_{x}^{\prime}+3 y\right) .
\end{aligned}
$$

In what follows, based on a mathematical construction recently developed in $[14,15]$ concerning the closed-form analytic solution of an Abel equation of the second kind of the normal form $y y_{x}^{\prime}-y=f(x)$, we provide the closed-form solutions of the reduced Abel equation (18), that is to say, the construction of the intermediate integral of the original TF equation (3) in the phase plane, as well as the final solution in the physical plane in accordance with the given boundary conditions.

\section{Closed-Form Solutions of the TF Equation in the Phase Plane-Final Solutions}

We consider the reduced Abel equation (18), namely, the equation

$$
u u_{\xi}^{\prime}-u=A \xi+B \xi^{3 / 2} ; \quad A=-\frac{12}{49}, B=\frac{1}{49}
$$

obeying (as the first of the boundary conditions (4) $y(0)=1$ and the well-known result $\left.y_{x}^{\prime}(0)=\stackrel{*}{B} \neq 0[2,8,17-20]\right)$ the transformed (19) and (20) equivalent condition:

$$
\text { at } \xi=\xi_{0}=0 \longrightarrow u(0)=u_{0}=0 \text {. }
$$

It was recently proved $[14,15]$ that an Abel equation of the second kind of the normal form $y y_{x}^{\prime}-y=f(x)$ admits an exact analytic solution in terms of known (tabulated) functions. Hence, we perform the solution of (21) which constitutes the solution of the TF equation in the phase plane as follows:

$$
\begin{aligned}
& \bar{\xi}=\ln |\xi+2 \stackrel{*}{C}|, \\
& u(\bar{\xi})=\frac{1}{2} \bar{\xi}\left[\bar{N}(\bar{\xi})+\frac{1}{3}\right], \\
& \bar{N}_{\bar{\xi}}^{\prime}(\bar{\xi})=\frac{4\left[G(\bar{\xi})+2\left(A \bar{\xi}+\bar{\xi}^{3 / 2}\right)\right]}{\bar{\xi}[\bar{N}(\bar{\xi})+4 / 3]}, \\
& \frac{1}{4} \frac{\left[(\bar{\xi} \sin \bar{\xi}+\cos \bar{\xi}) \stackrel{*}{A}(\bar{\xi})+\cos ^{2} \bar{\xi}\right]\left(2 \bar{\xi}^{*} A(\bar{\xi})+\cos \bar{\xi}\right)}{\left(\bar{\xi}^{*} A(\bar{\xi})\right)^{3} e^{\bar{\xi}}} \\
& =4\left[\bar{G}+2\left(A \bar{\xi}+B \bar{\xi}^{3 / 2}\right)\right] \text {, }
\end{aligned}
$$


where

$$
\begin{aligned}
\stackrel{*}{A} & =\stackrel{*}{A}(\bar{\xi})=\text { the cosine integral }=\operatorname{ci}(\bar{\xi}) \\
& =\mathbb{C}+\ln \bar{\xi}+\sum_{u=1}^{\infty}(-1)^{u} \frac{\bar{\xi}^{2 u}}{(2 u)(2 u) !} ; \\
& \mathbb{C}=\text { Euler's number }=0,5572156649015325 \ldots
\end{aligned}
$$

Here $\stackrel{*}{C}$ is integration constant, while the function $\bar{N}(\bar{\xi})$ is given implicitly in terms of the subsidiary function $G(\bar{\xi})$, which can be determined from (23b), and the known member of (21) as in the following three cases.

Case $1(Q(\bar{\xi})<0(p<0))$. Consider

$$
\begin{aligned}
\bar{N}^{(1)}(\bar{\xi}) & =2 \sqrt{-\frac{p}{3}} \cos \frac{a}{3}, \\
\bar{N}^{(2)}(\bar{\xi}) & =-2 \sqrt{-\frac{p}{3}} \cos \frac{a-\pi}{3}, \\
\bar{N}^{(3)}(\bar{\xi}) & =-2 \sqrt{-\frac{p}{3}} \cos \frac{a+\pi}{3} ; \\
\cos a & =-\frac{q}{2 \sqrt{-(p / 3)^{3}}} ; \quad 0<a<\pi .
\end{aligned}
$$

Case $2(Q(\bar{\xi})>0)$. Consider

$$
\bar{N}(\bar{\xi})=\sqrt[3]{-\frac{q}{2}+\sqrt{Q}}+\sqrt[3]{-\frac{q}{2}-\sqrt{Q}} .
$$

Case $3(Q(\bar{\xi})=0)$. Consider

$$
\begin{aligned}
& \bar{N}^{(1)}(\bar{\xi})=2 \sqrt[3]{-\frac{q}{2}}, \\
& \bar{N}^{(2)}(\bar{\xi})=\bar{N}^{(3)}(\bar{\xi})=-\sqrt[3]{-\frac{q}{2}} .
\end{aligned}
$$
by

In all these formulae the quantities $Q, p$, and $q$ are given

$$
\begin{aligned}
Q(\bar{\xi}) & =\frac{1}{27} p^{3}(\bar{\xi})+\frac{1}{4} q^{2}(\bar{\xi}) ; \\
p(\bar{\xi}) & =-\frac{a^{2}}{3}+b, \\
q(\bar{\xi}) & =\frac{2}{27} a^{3}-\frac{1}{3} a b+c ; \\
a & \\
b & =\frac{3+4\left[G(\bar{\xi})+\left(A \bar{\xi}+B \bar{\xi}^{3 / 2}\right)\right]}{\bar{\xi}}, \\
c & =\frac{4\left[G(\bar{\xi})+2\left(A \bar{\xi}+B \bar{\xi}^{3 / 2}\right)\right]}{\bar{\xi}} .
\end{aligned}
$$

In order to define the type of the function $\bar{N}^{(i)}(\bar{\xi})$ $(i=1,2,3)$ which must be selected among the closed-form solutions ((23a), (23b), and (23c)) to (26), as well as the value of the subsidiary function $G(\bar{\xi})$ at $\bar{\xi}=0$ and the value of the integration constant $\stackrel{*}{C}$, we must combine the above Abel solutions with the boundary data.

We symbolize by $\bar{\xi}=\ln \left|2^{*}\right|$ the substitution while by $\mathscr{L}_{0}$ any function $\mathscr{L}(\bar{\xi})$ at $\bar{\xi}_{0}=\ln |2 \bar{C}|$. Thus functions $u(\bar{\xi})$ and $\left(\bar{N}_{\bar{\xi}}^{\prime}\right)(\bar{\xi})$ given in (23a) perform

$$
\begin{aligned}
& u(\bar{\xi})=\frac{1}{2} \bar{\xi}\left[\bar{N}^{(i)}(\bar{\xi})+\frac{1}{3}\right], \\
& \bar{N}_{\bar{\xi}}^{(i)}(\bar{\xi})=\frac{4\left[G(\bar{\xi})+2\left(-(12 / 49)(\bar{\xi})+(1 / 49) \bar{\xi}^{3 / 2}\right)\right]}{\bar{\xi}\left[\bar{N}^{(i)}(\bar{\xi})+4 / 3\right]}, \\
& u_{0}=\frac{1}{2} \ln |2 C|\left(\bar{N}_{0}^{(i)}+\frac{1}{3}\right) \text {, } \\
& \bar{N}_{\bar{\xi}_{0}}^{\prime(i)}(\bar{\xi}) \\
& =\frac{4\left[G_{0}+2\left(-(12 / 49) \ln |2 \dot{C}|+(1 / 49)(\ln |2 \dot{C}|)^{3 / 2}\right)\right]}{\ln |2 \dot{C}|\left[\bar{N}_{0}^{(i)}+4 / 3\right]} .
\end{aligned}
$$

On the other hand, the Abel nonlinear ODE (21) at $\bar{\xi}_{0}$ becomes

$$
\begin{aligned}
1+ & \frac{-(12 / 49) \ln \left|2{ }^{*}\right|+(1 / 49)\left(\ln \left|2 C^{*}\right|\right)^{3 / 2}}{u_{0}} \\
& =1+\frac{-(12 / 49) \ln \left|2 C^{*}\right|+(1 / 49)\left(\ln \left|2 C^{*}\right|\right)^{3 / 2}}{\ln \left|22^{*}\right|\left(\bar{N}_{0}^{(i)}+4 / 3\right)} \\
& =\frac{u_{\bar{\xi}_{0}}^{\prime}}{\bar{\xi}_{\xi_{0}}^{\prime}} .
\end{aligned}
$$

Combination of the above equations results in the following quadratic to $\bar{N}_{0}$ equation:

$$
\begin{aligned}
& \bar{N}_{0}^{(i)^{2}}+\bar{N}_{0}^{(i)}+\frac{4}{9}+\left[1-\frac{1}{\dot{C} \ln \left|22^{*}\right|}\right] \\
& \cdot\left[-2 G_{0}+2\left(-\frac{12}{49} \ln \left|2 \stackrel{C}{C}^{*}\right|+\frac{1}{49}\left(\ln \left|2{ }^{*}\right|\right)^{3 / 2}\right)\right] \\
& =0
\end{aligned}
$$

including the value of $G_{0}$ and the constant on integration ${ }^{*}$. 
Similarly, by (23b) one gets

$$
\begin{aligned}
& 32\left\{G_{0}+\left[2\left(-\frac{12}{49} \ln |2 \stackrel{*}{C}|+\frac{1}{49} \ln |2 \stackrel{*}{C}|^{3 / 2}\right)\right]{\stackrel{*}{A^{3}}}_{0}\right. \\
& =\left\{\left[\ln |2 \stackrel{*}{C}| \sin \left(\ln \left|2^{*}\right|\right)+\cos (\ln |2 \stackrel{*}{C}|)\right]^{\bar{A}_{0}}\right. \\
& \left.+\cos ^{2}(\ln |2 \stackrel{*}{C}|)\right\}\left(2 \ln |2 \stackrel{*}{C}| \stackrel{*}{A}_{0}\right. \\
& +\cos (\ln |2 \stackrel{*}{C}|))
\end{aligned}
$$

that constitute a second equation including the value of $G_{0}$

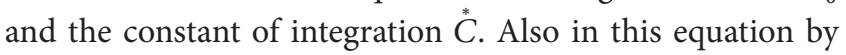
means of $(23 \mathrm{c})$ we have

$$
\begin{aligned}
& \stackrel{*}{A}_{0}= \stackrel{*}{A}(\ln |2 \stackrel{*}{C}|)=\operatorname{ci}\left(\ln \left|2^{*}\right|\right) \\
&=\mathbb{C}+\ln (\ln |2 \stackrel{C}{C}|)+\sum_{k=1}^{\infty}(-1)^{k} \frac{\ln \left|2 \mathscr{C}^{2}\right|^{2 k}}{(2 k)(2 k) !} \\
& \mathbb{C}=\text { Euler's number } \approx 0.5572 .
\end{aligned}
$$

Summarizing, from (19) and (20) one concludes that, since by the first boundary conditions $y(0)=1$ for $x \rightarrow 0 \Rightarrow y \rightarrow 1$ then for $x \rightarrow 0 \Rightarrow y_{x}^{\prime} \rightarrow 0$ or $y_{x}^{\prime} \rightarrow \stackrel{*}{B}$, where ${ }_{B}^{*}$ is a fixed value. Thus, combining (23a), (23b), and (23c) together with the first of (28) and the Abel nonlinear ODE (21), one may write that, for $x \rightarrow 0 \Rightarrow \xi_{0} \rightarrow 0, u_{0} \rightarrow 0, \bar{N}_{0} \rightarrow 0$. Therefore, by making use of the first boundary condition given in (4) $(x \rightarrow 0, y \rightarrow 1$,$) as well as of all the above$ observations, the solutions of (31) and (32) perform the values for $G_{0}$ and $2{ }^{*}$ and they can be written in the following algebraic forms:

$$
\begin{aligned}
\frac{-1 \pm \sqrt{1-4 \mathscr{F}\left(\bar{G}_{0}, \stackrel{*}{C}\right)}}{2} & =0 \\
\mathscr{H}(\stackrel{*}{C}) & =\bar{G}_{0},
\end{aligned}
$$

where

$$
\begin{aligned}
& \mathscr{F}\left(G_{0}, \stackrel{*}{C}\right)=\frac{4}{9}+\left[1-\frac{1}{\stackrel{*}{C} \ln |2 \stackrel{*}{C}|}\right]\left[-2 G_{0}\right. \\
& \left.+2\left(-\frac{12}{4} \ln \left|2^{*}\right|+\frac{1}{49}\left(\ln \left|2^{*}\right|\right)^{3 / 2}\right)\right] ;
\end{aligned}
$$

$$
\begin{aligned}
& \mathscr{H}(\stackrel{*}{C}) \\
& =\frac{1}{32}\left\{\left[\ln \left|2^{*}\right| \sin \left(\ln \left|2{ }^{C}\right|\right)+\cos \left(\ln \left|2 \stackrel{*}{C}^{C}\right|\right)\right]\right. \\
& \left.\cdot \bar{*}_{00}+\cos ^{2}(\ln |2 \stackrel{*}{C}|)\right\}\left[2 \ln |2 \stackrel{*}{C}| \bar{*}_{0}\right. \\
& +\cos (\ln |2 \stackrel{*}{C}|)] \\
& \bar{*}_{0}, \stackrel{*}{C} \text { as in }(33) \text {. }
\end{aligned}
$$

Thus, we are now able to estimate $p_{0}, q_{0}$, and $Q_{0}$ given by (27) in terms of the known values $\stackrel{*}{C}$ and $G_{0}$. This estimation furnishes the value of $Q_{0}$ and therefore the type of the solution $\bar{N}^{(i)}(\xi)(i=1,2,3)$ near the origin $\xi_{0}$ among the formulae (24) to $(26)$.

From the above evaluation, it is concluded that the closed form solutions of the Abel nonlinear ODE (21) given as

$$
\begin{aligned}
\bar{u}^{(i)}(\bar{\xi}) & =\frac{1}{2} \bar{\xi}\left[\bar{N}^{(i)}(\bar{\xi})+\frac{1}{3}\right] \\
\bar{\xi} & =\ln \left|\xi+2 \stackrel{*}{C}_{1}\right|
\end{aligned}
$$

$$
(i=1,2,3)
$$

have been completely determined.

Since we obtained the above closed-form solutions of the intermediate Abel nonlinear ODE (21) the solutions to the original TF equation (3) can be determined as follows. By (19) and (21) we perform

$$
\begin{aligned}
& x=\frac{1}{7^{1 / 3}}\left(\frac{\xi}{y}\right)^{1 / 3}, \\
& y_{x}^{\prime}=\frac{1}{x^{4}} u-3 \frac{y}{x},
\end{aligned}
$$

while taking the total differential in (19) we extract

$$
\begin{aligned}
d \xi & =21 x^{2} y d x+7 x^{3} d y \\
& \Longleftrightarrow \xi_{x}^{\prime}=\frac{d \xi}{d x}=7 x^{2}\left(x y_{x}^{\prime}+3 y\right)=7 \frac{u}{x}
\end{aligned}
$$

where $u=u\left(\xi+2{ }^{*}{ }_{1}\right)$ denotes the known solution of the Abel nonlinear ODE (18). Using (34a), (34b), and (37) one writes

$$
\begin{aligned}
y_{\xi}^{\prime} & =\frac{y_{x}^{\prime}}{\xi_{x}^{\prime}}=7^{4 / 3}\left(\frac{y}{\xi}\right) u^{4 / 3}-3 y 7^{1 / 3}\left(\frac{y}{\xi}\right)^{1 / 3} \\
& =\frac{u(\xi)}{\xi} y-\frac{3}{7 \xi^{4 / 3}} y^{4 / 3} ;
\end{aligned}
$$


that is, the Bernoulli equation

$$
\begin{array}{r}
y^{1 / 3}=\frac{\exp \left((1 / \xi) \int(u(\xi) / \xi) d \xi\right)}{C+(1 / 7) \int \exp \left[\left((1 / \xi) \int(u(\xi) / \xi) d \xi\right)\right] d \xi}, \\
x=\frac{1}{7^{1 / 3}}\left(\frac{\xi}{y}\right)^{1 / 3} ; \\
x \geq 0, y \geq 0, \xi=\text { parameter }(\xi \geq 0) ; \\
C=\text { second constant of integration. }
\end{array}
$$

The last step of the above analysis concerns the calculation of the second constant of integration $C$. Thus from the first of the boundary conditions (4) and the parametric solution (40), one writes

$$
\begin{aligned}
& x_{1} \longrightarrow 0, \\
& y_{1} \longrightarrow 1, \\
& \xi_{1} \longrightarrow 0, \\
& \lim _{\xi_{1} \rightarrow 0}\left(y^{1 / 3}\right) \\
& \quad=\frac{\lim _{\xi_{1} \rightarrow 0}\left\{\exp \left((1 / \xi) \int(u(\xi) / \xi) d \xi\right)\right\}}{\lim _{\xi_{1} \rightarrow 0}\left\{C+(1 / 7) \int \exp \left[\left((1 / \xi) \int(u(\xi) / \xi) d \xi\right)\right] d \xi\right\}}
\end{aligned}
$$

and estimates the constant of integration $C$. By the second of the boundary conditions (4) one writes $x_{2} \rightarrow+\infty, y_{2} \rightarrow 0$, and $\xi \rightarrow \xi_{2}$. Again through the parametric solution (40) one writes

$$
\lim _{\xi \rightarrow \xi_{2}}\left[\exp \left[\frac{1}{\xi} \int \frac{u(\xi)}{\xi} d \xi\right]\right]
$$

and evaluates the value of $\xi_{2}$. The evaluation of the second constant of integration can be obtained again through the boundary condition $1 / x_{1} \rightarrow 0 \Rightarrow y_{1} \rightarrow \infty$; that is,

$$
\begin{aligned}
& \lim _{\xi \rightarrow \xi_{1}}\left\{C+\frac{1}{7} \int \exp \left[\left(-\frac{1}{\xi} \int \frac{u(\xi)}{\xi} d \xi\right) \frac{1}{\xi^{1 / 3}} d \xi\right]\right\} \\
& \quad=0
\end{aligned}
$$

Equations (41) and (42a), (42b) complete the solutions of the problem under consideration that is the construction of a closed-form parametric solution of the TF equation (3).

It is worthwhile to remark that since the solution is given in a closed form the mathematical methodology being developed results in the ad hoc definition of the TF function $y$ in approximate power series with $y_{x}^{\prime}(0)=\stackrel{*}{B}(\stackrel{*}{B}=$ a factor that must be determined) and with boundary conditions given by $y_{0}(0)=x_{0}=1 ; y_{1} \rightarrow 0$ as $x \rightarrow \infty$. The value $y_{x}^{\prime}(0)=\stackrel{*}{B}$ can be evaluated by way of the already constructed closedform solution. This is a very interesting result in accordance with previous results presented by Bush and Caldwell [17], Feynman et al. [18], Coulson and March [19], Kobayashi et al. [20], and Kobayashi [21].

\section{Closed-Form Solutions of the LB Equation in the Physical Plane}

It was already proved (Section 3) that the LB nonlinear ODE (1) has been reduced to an Abel ODE of the second kind of the normal form (15). It was also proved that this Abel equation admits exact analytic solutions given, as in case of the TF nonlinear ODE, by (24) to (26). Thus, the solution of the LB equation (1) in the phase plane is given by the formulae (24) to (26), if instead of the parenthesis $(A \xi+$ $\left.B \xi^{3 / 2}\right)$, the quantity $\left(\xi^{-1 / 2} / 4-\xi / 4\right)$ is introduced. The solution of the original equation (1) in the physical plane is given through the combination of the above prescribed formulae in combination with (17). As in case of the TF equation, the whole problem includes two constants of integration $\stackrel{*}{C}^{*}$ and $C$, which are to be determined by using the convenient boundary data of the problem under consideration. In order to define simultaneously the type of the function $\bar{N}^{(i)}(\bar{\xi})$ $(i=1,2,3)$ which must be selected in accordance with the modified previously developed formulae (24) to (26) and thus define the modified function $G(\bar{\xi})$ and the constant of integration $\stackrel{*}{C}$, we must combine the above Abel solutions with the prescribed convenient boundary data.

Based on what was previously developed in Section 3 for the original (LB) equation (1), the following boundary conditions hold true:

$$
\text { at } \begin{aligned}
x & =x_{0} \\
& \Longrightarrow y=y_{0}=\ell, \\
y_{x}^{\prime} & =y_{x_{0}}^{\prime}=m,
\end{aligned}
$$

where $\ell$ and $m$ are arbitrary given values provided $m \neq 0$.

Based on these boundary conditions, the substitution (8) furnishes $p\left(y_{0}\right)=p_{0}=m$, while transformation (10) furnishes $w\left(y_{0}\right)=w_{0}=y_{0}^{1 / 3} p_{0}=\ell^{1 / 3} m$ too. Thus, the substitutions (13), (15) and the Abel nonlinear ODE (16) perform the following relations:

$$
\begin{aligned}
w_{0} & =n\left(\xi_{0}\right)=n_{0}=\ell^{1 / 3} m, \\
\xi_{0} & =y_{0}^{4 / 3}=\ell^{4 / 3} ; \\
n_{0} & =-z\left(\xi_{0}\right)=-z_{0} \\
& \Longrightarrow z_{0}=-\ell^{1 / 3} m ; \\
z_{\xi_{0}}^{\prime} & =-\frac{(1 / 4)\left(\ell^{-2 / 3}-\ell^{4 / 3}\right)}{\ell^{1 / 3} m}+1=1-\frac{1}{4} \frac{\ell^{-1}-\ell}{m} .
\end{aligned}
$$

We are now able to evaluate all the before mentioned quantities. Indeed, (23a) and (23b) for $\xi_{0}=\ell^{4 / 3}, z_{0}=-\ell^{1 / 3} m$, 


$$
\begin{aligned}
& z_{\xi_{0}}^{\prime}=1-\left(\ell^{-1}-\ell\right) / 4 m \text { furnish the following two equations: } \\
& \bar{N}_{0}=-\left(\frac{2 \ell^{1 / 3} m}{\ell^{4 / 3}+4 C}+\frac{1}{3}\right) \\
& \frac{8 G_{0}+2\left(\ell^{4 / 3}-\ell^{1 / 3}\right) \mathfrak{P} \ln |\mathfrak{P}|\left[\operatorname{ci}(\ln |\mathfrak{P}|)-\ln |\mathfrak{P}|+\ln (\ln |\mathfrak{P}|)-\ln \left(\ln \left|1-2 C^{*}\right|\right)-\mathbb{C}\right]}{4\left[\operatorname{ci}(\ln |\mathfrak{P}|)-\ln |\mathfrak{P}|+\ln (\ln |\mathfrak{P}|)-\ln \left(\ln \left|1-2 C^{*}\right|\right)-\mathbb{C}\right]-\cos (\ln |\mathfrak{P}|)} \\
& =\frac{1}{4} \frac{\left[\operatorname{ci}(\ln |\mathfrak{P}|)-\ln |\mathfrak{P}|+\ln (\ln |\mathfrak{P}|)-\ln \left(\ln \left|1-2 C^{*}\right|\right)-\mathbb{C}\right][\ln |\mathfrak{P}| \sin (\ln |\mathfrak{P}|)+\cos (\ln |\mathfrak{P}|)]}{[\operatorname{ci}(\ln |\mathfrak{P}|)-\ln |\mathfrak{P}|+\ln (\ln |\mathfrak{P}|)-\ln (\ln |1-2 C|)-\mathbb{C}]^{2}(\ln |\mathfrak{P}|)^{2}} \\
& +\frac{\cos ^{2}(\ln |\mathfrak{P}|)}{[\operatorname{ci}(\ln |\mathfrak{P}|)-\ln |\mathfrak{P}|+\ln (\ln |\mathfrak{P}|)-\ln (\ln |1-2 \mathfrak{C}|)-\mathbb{C}]^{2}(\ln |\mathfrak{P}|)^{2}}
\end{aligned}
$$

where $\bar{N}_{0}$ and $G_{0}$ are the values of $\bar{N}(\bar{\xi})$ and $G(\bar{\xi})$ at $\xi=\xi_{0}=$ $\ell^{4 / 3}$, respectively. On the other hand, the formula for $\bar{N}_{\bar{\xi}}^{\prime}$ in (23a) at $\xi=\xi_{0}$ results in

$$
\bar{N}_{\xi_{0}}^{\prime}=\frac{1}{\left(\bar{N}_{0}+4 / 3\right)} \frac{4\left[G_{0}+(1 / 2)\left(\ell^{-2 / 3}-\ell^{4 / 3}\right)\right]}{\left(\ell^{4 / 3}+4 \stackrel{*}{C}\right)^{2}},
$$

while by the first of (23a) and (44) we have

$$
z_{\xi_{0}}^{\prime}=\frac{1}{2} \bar{N}_{\xi_{0}}^{\prime}\left(\xi_{0}+4 \stackrel{*}{C}\right)+\frac{1}{2}\left(\bar{N}_{0}+\frac{1}{3}\right),
$$

or equivalently

$$
z_{\xi_{0}}^{\prime}=\frac{2\left[G_{0}+(1 / 2)\left(\ell^{-2 / 3}-\ell^{4 / 3}\right)\right]}{\left(\bar{N}_{0}+4 / 3\right)\left(\ell^{4 / 3}+4 C\right)}+\frac{1}{2}\left(\bar{N}_{0}+\frac{1}{3}\right) .
$$

The last relation (48) in combination together with (44) permits us to write

$$
\begin{aligned}
& \frac{2\left[G_{0}+(1 / 2)\left(\ell^{-2 / 3}-\ell^{4 / 3}\right)\right]}{\left(\bar{N}_{0}+(4 / 3)\right)\left(\ell^{4 / 3}+4 \mathrm{C}\right)}+\frac{1}{2}\left(\bar{N}_{0}+\frac{1}{3}\right) \\
& \quad=1-\frac{1}{4} \frac{\ell^{-1}-\ell}{m} .
\end{aligned}
$$

The above three equations (46) to (49) constitute a nonlinear (transcendental) system for $\bar{N}_{0}, G_{0}$, and $\lambda$ by means of which one calculates these unknowns in terms of the parameters $\ell$ and $m$. Thus, the discriminant $Q_{0}\left(Q\right.$ at $\left.\xi=\xi_{0}=\ell^{4 / 3}\right)$ given in (27) is also known, the fact that permits us to define the type of the function $\bar{N}^{(i)}(\bar{\xi})$ for $\xi>\xi_{0}=\ell^{4 / 3}$ (24) to (26). This completes the solution of the problem under consideration,

$$
\mathfrak{P}=\ell^{4 / 3}+2^{*} \mathrm{C}>0,2^{*}+l^{4 / 3}>0,2^{*}<1,
$$

which is the construction of the solution of the LB equation in the phase-plane (and thus in the physical plane) in the main interval $\left[\xi_{0}=\ell^{4 / 3}, \bar{\xi}\right]$. If this solution is not unique inside $\left[\ell^{4 / 3}, \bar{\xi}\right]$, then we follow step by step the methodology developed in [15].

\section{Conclusions}

By a series of admissible functional transformations we reduce the nonlinear TF and LB equations to Abel's equations of the second kind of the normal form of (16) and (18), respectively. These equations do not admit closed-form solutions in terms of known (tabulated) functions. This unsolvability is due to the fact that only very special forms of this kind of (16) and (18) can be solved in parametric form [4]. Our goal is the development of the construction of the exact analytic solutions of the above equations based on a mathematical technique leading to the derivation of close form solutions for the Abel equation of the second kind of the normal form $[3,4]$. The proposed methodology constitutes the intermediate integral of the TF and LB equations in the phase and physical planes. The reduction procedure in the paper and the constructed solutions are very general and can be applied to a large number of nonlinear ODEs in mathematical physics and nonlinear mechanics. Also the method employed is exact in accordance with the boundary conditions of each problem under consideration and guides us to the a priori approximate construction of the solutions in power series, some coefficients of which must be evaluated.

With the proposed procedure the exact value of first order derivative of TF equation is derived at $x=0$. In particular, the solution for the TF equation is finally constructed by calculating 4 constants from the specific boundary conditions (4) (see (34a), (34b), (41), (42a), and (42b)). Equations (40) constitute the parametric solutions of the TF problem. With our method essentially a different way of calculating 
$y_{x}^{\prime}(0)={ }_{B}^{*}$ (Section 4$)$ is proposed with reference to the methods proposed in [17-21], where the value of ${ }_{B}^{*}$ has been calculated having kept the solution from approximate series in which their coefficients had been calculated from the boundary conditions. In conclusion, in this paper an analytic method is developed that does not contradict previous results but it is a different way of calculating them.

\section{Conflict of Interests}

The authors declare that there is no conflict of interests regarding the publication of this paper.

\section{References}

[1] I. Langmuir and K. B. Blodgett, "Currents limited by space charge between coaxial cylinders," Physical Review, vol. 22, no. 4, pp. 347-356, 1923.

[2] H. T. Davis, Introduction to Nonlinear Differential and Integral Equations, Dover, New York, NY, USA, 1962.

[3] E. Kamke, Differentialgleichungen I, Gewöhnliche Differentialgleichungen and II, Partielle Differentialgleichungen, Akademische Verlagsgesellschaft, Leipzig, Germany, 1962.

[4] A. D. Polyanin and V. F. Zaitsev, Handbook of Exact Solutions for Ordinary Differential Equations, CRC Press, Boca Raton, Fla, USA, 1999.

[5] K. Horváth, J. N. Fairchild, K. Kaczmarski, and G. Guiochon, "Martin-Synge algorithm for the solution of equilibriumdispersive model of liquid chromatography," Journal of Chromatography A, vol. 1217, no. 52, pp. 8127-8135, 2010.

[6] Y. Shang and X. Zheng, "The first-integral method and abundant explicit exact solutions to the zakharov equations," Journal of Applied Mathematics, vol. 2012, Article ID 818345, 16 pages, 2012.

[7] D. B. Graham and I. H. Cairns, "Constraints on the formation and structure of langmuir eigenmodes in the solar wind," Physical Review Letters, vol. 111, no. 12, Article ID 121101, 2013.

[8] L. H. Thomas, "The calculation of atomic fields," Mathematical Proceedings of the Cambridge Philosophical Society, vol. 23, no. 5, pp. 542-548, 1927.

[9] E. Fermi, "Statistical method of investigating electrons in atoms," Zeitschrift für Physik, vol. 48, pp. 73-79, 1927.

[10] T. Tsurumi and M. Wadati, "Dynamics of magnetically trapped boson-fermion mixtures," Journal of the Physical Society of Japan, vol. 69, no. 1, pp. 97-103, 2000.

[11] S. Liao, "An explicit analytic solution to the Thomas-Fermi equation," Applied Mathematics and Computation, vol. 144, no. 2-3, pp. 495-506, 2003.

[12] S. Abbasbandy and C. Bervillier, "Analytic continuation of Taylor series and the boundary value problems of some nonlinear ordinary differential equations," Applied Mathematics and Computation, vol. 218, no. 5, pp. 2178-2199, 2011.

[13] K. Ourabah and M. Tribeche, "Relativistic formulation of the generalized nonextensive Thomas-Fermi model," Physica A, vol. 393, pp. 470-474, 2014.

[14] D. E. Panayotounakos, "Exact analytic solutions of unsolvable classes of first and second order nonlinear ODEs (part I: Abel's equations)," Applied Mathematics Letters, vol. 18, no. 2, pp. 155$162,2005$.
[15] D. E. Panayotounakos, N. B. Sotiropoulos, A. B. Sotiropoulou, and N. D. Panayotounakou, "Exact analytic solutions of nonlinear boundary value problems in fluid mechanics (Blasius equations)," Journal of Mathematical Physics, vol. 46, no. 3, Article ID 033101, 2005.

[16] D. E. Panayotounakos, E. E. Theotokoglou, and M. P. Markakis, "Exact analytic solutions for the unforded damped duffing nonlinear oscillator," Comptes Rendus Mechanics Journal, vol. 334, pp. 311-316, 2006.

[17] V. Bush and S. H. Caldwell, "Thomas-Fermi equation solution by the differential analyzer," Physical Review, vol. 38, no. 10, pp. 1898-1901, 1931.

[18] R. P. Feynman, N. Metropolis, and E. Teller, "Equations of state of elements based on the generalized fermi-thomas theory," Physical Review, vol. 75, no. 10, pp. 1561-1573, 1949.

[19] C. A. Coulson and N. H. March, "Momenta in atoms using the Thomas-Fermi method," Proceedings of the Physical Society Section A, vol. 63, no. 4, pp. 367-374, 1950.

[20] S. Kobayashi, T. Matsukuma, S. Nagai, and K. Umeda, "Accurate value of the initial slope of the ordinary TF function," Journal of the Physical Society of Japan, vol. 10, no. 9, pp. 759-762, 1955.

[21] S. Kobayashi, "Some coefficients of the series expansion of the TFD function," Journal of the Physical Society of Japan, vol. 10, no. 9, pp. 824-825, 1955.

[22] S. A. Sommerfeld, "Integrazione Asintotica dell'Equazione Differenziali di Thomas-Fermi," Accademia dei Lincei, Atti-Rendiconte, vol. 4, pp. 85-110, 1932. 


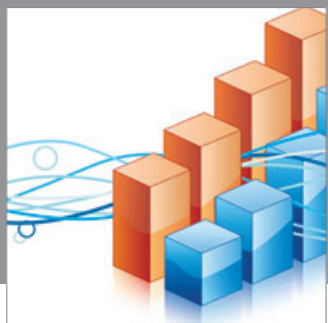

Advances in

Operations Research

mansans

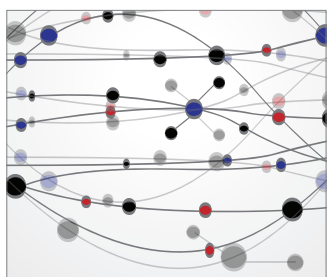

The Scientific World Journal
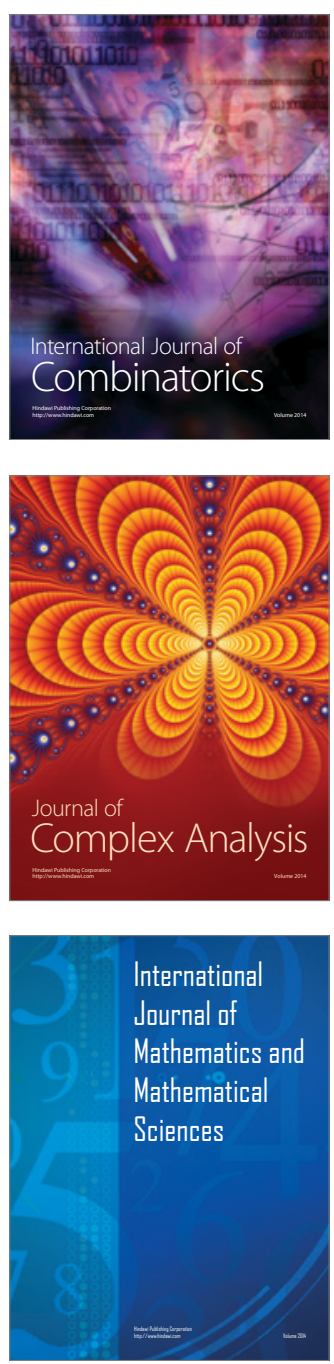
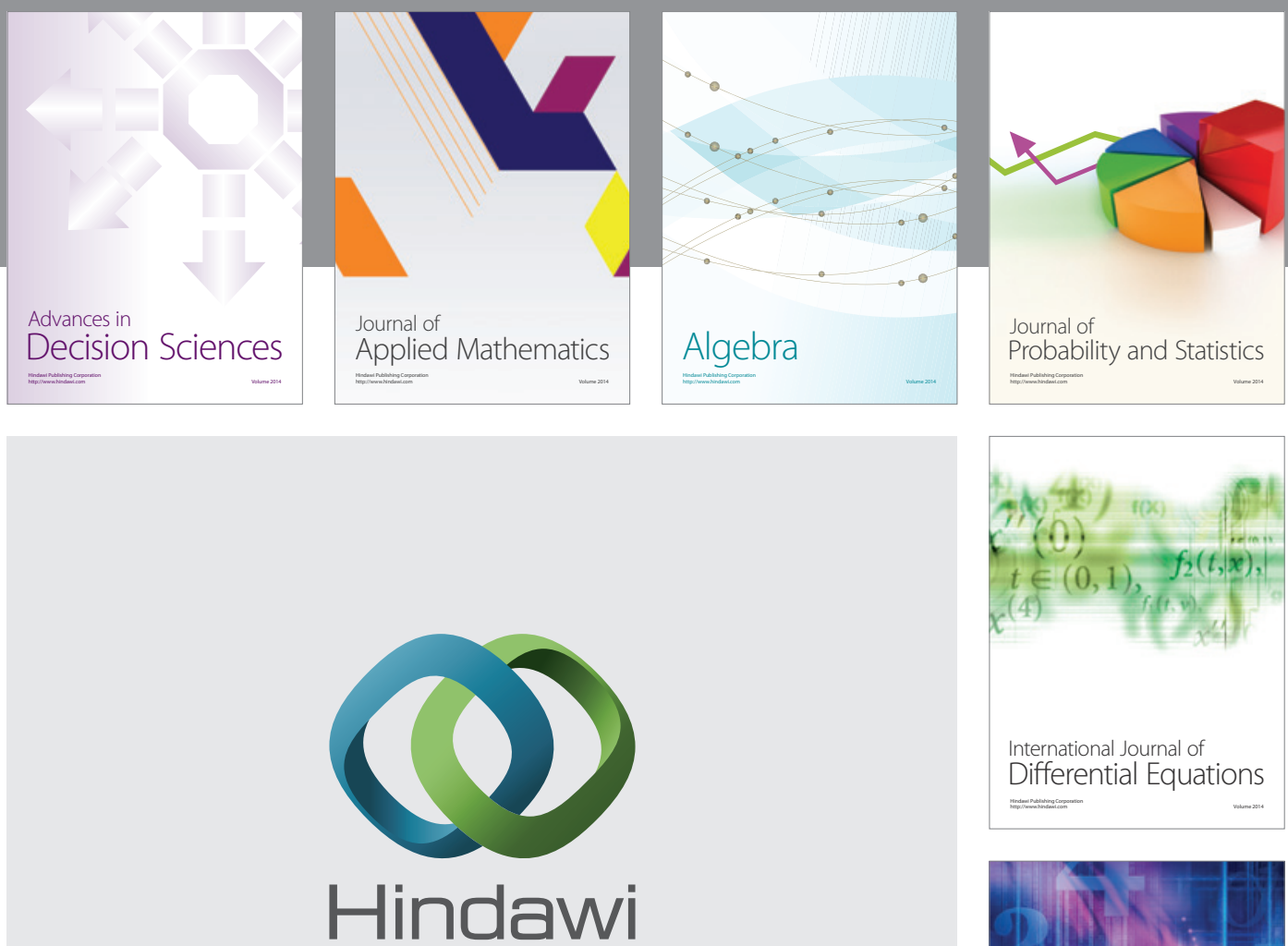

Submit your manuscripts at http://www.hindawi.com
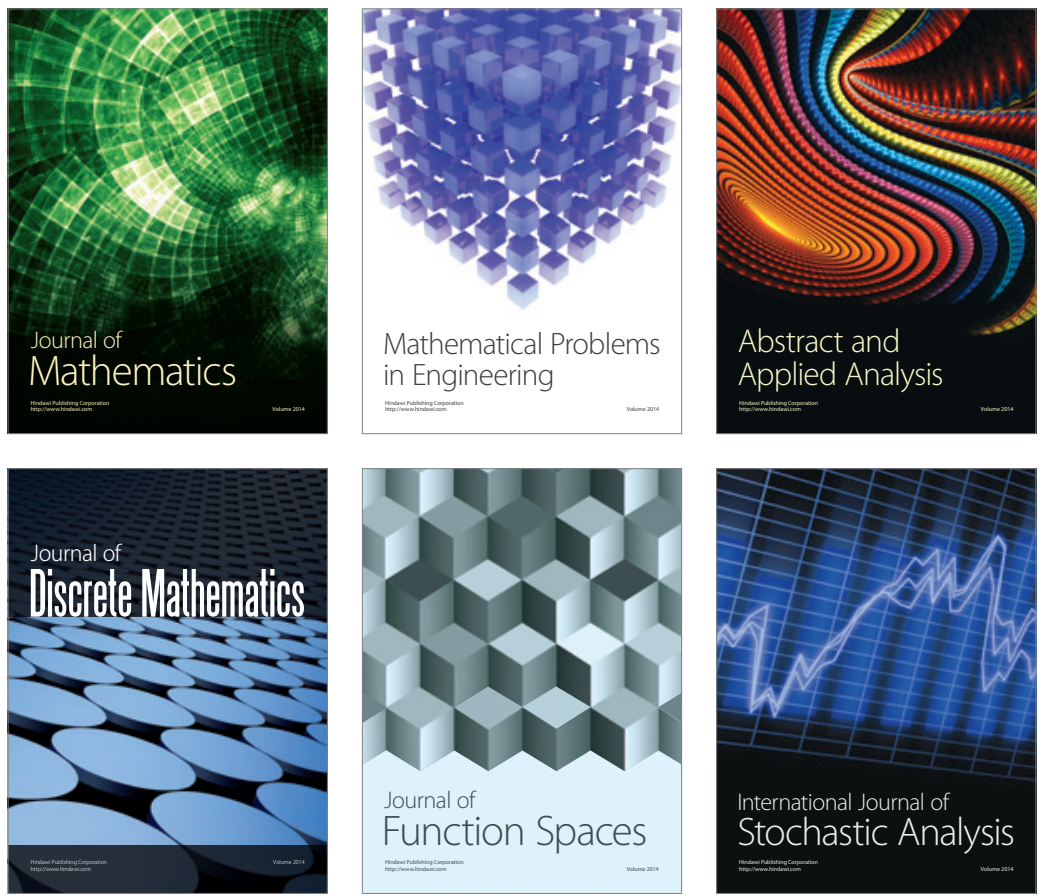

Journal of

Function Spaces

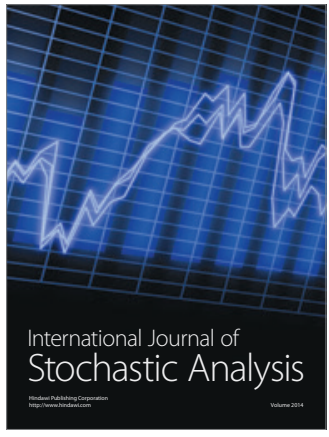

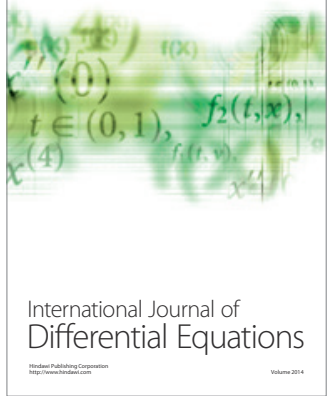
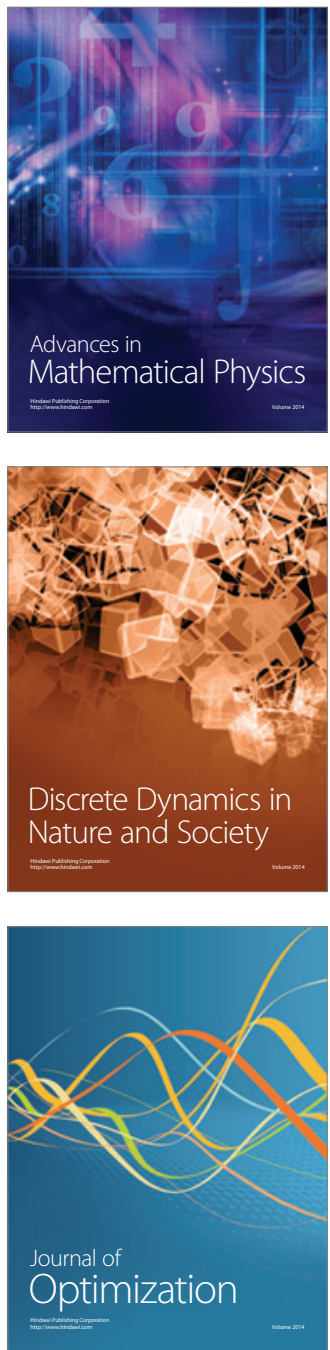